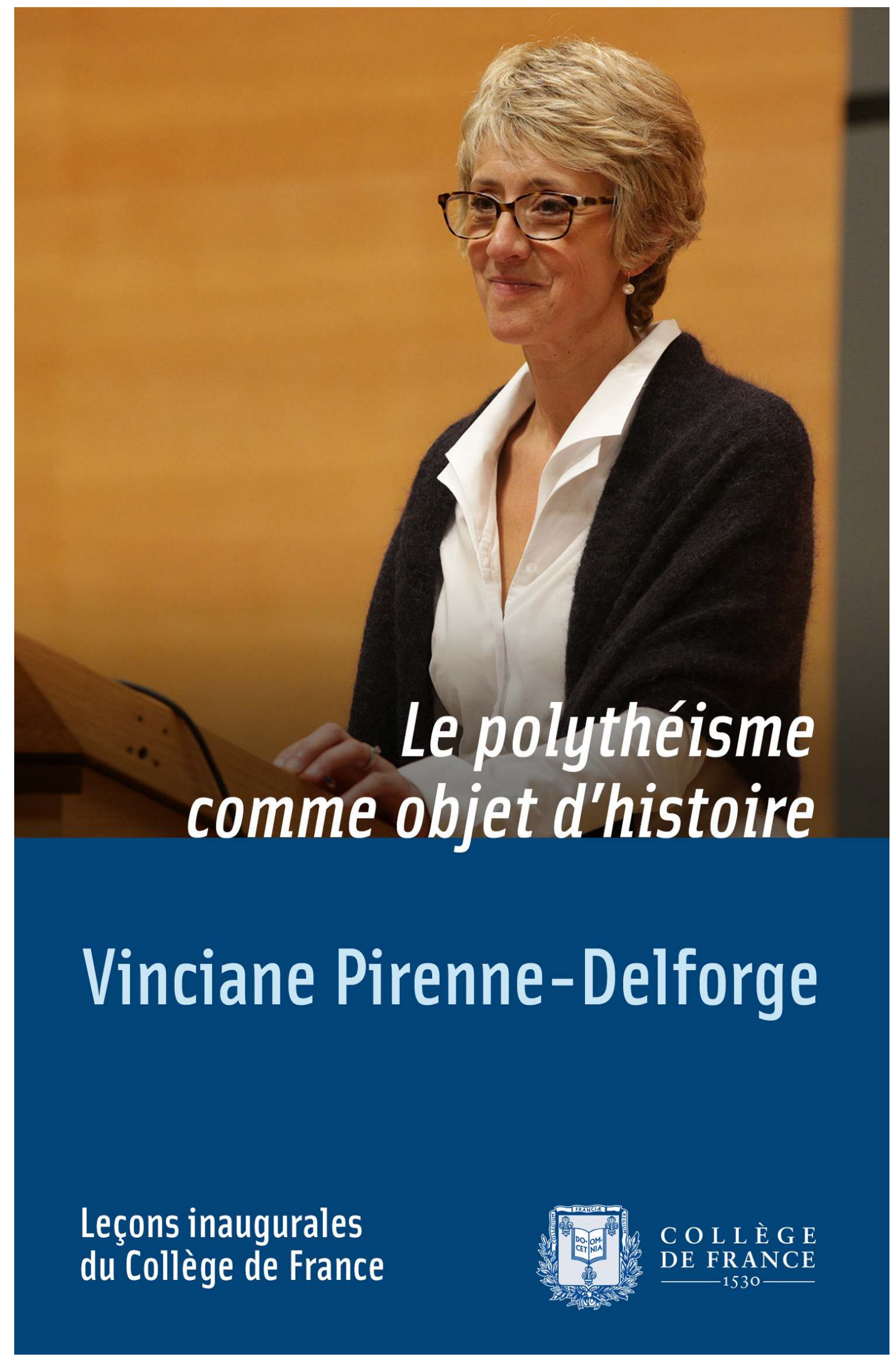




\section{Le polythéisme grec comme objet d'histoire}

Leçon inaugurale prononcée le jeudi 7 décembre 2017

\section{Vinciane Pirenne-Delforge}

Éditeur : Collège de France

Lieu d'édition : Paris

Année d'édition : 2018

Date de mise en ligne : 20 juin 2019

Collection : Leçons inaugurales

ISBN électronique : 9782722604933

\section{opentatition ebooks}

http://books.openedition.org

Édition imprimée

Date de publication : 6 juin 2018

ISBN : 9782213709727

Nombre de pages : 64

Ce document vous est offert par Collège de France

COLLEGE

DE FRANCE

Référence électronique

PIRENNE-DELFORGE, Vinciane. Le polythéisme grec comme objet d'histoire : Leçon inaugurale prononcée le jeudi 7 décembre 2017. Nouvelle édition [en ligne]. Paris : Collège de France, 2018 (généré le 21 juin 2019). Disponible sur Internet : <http://books.openedition.org/cdf/6159>. ISBN : 9782722604933.

Ce document a été généré automatiquement le 21 juin 2019.

(c) Collège de France, 2018

Conditions d'utilisation

http://www.openedition.org/6540 
Le polythéisme grec n'est pas une simple juxtaposition de divinités, mais il est fait de leurs interactions à différentes échelles - locale, régionale, panhellénique. De précieuses moissons documentaires - combinant sources littéraires, épigraphiques, archéologiques, iconographiques - en ont profondément renouvelé l'étude et l'ont constitué en objet pertinent pour comprendre les sociétés anciennes dans une perspective tant historique qu'anthropologique. La religion grecque étant profondément imbriquée dans des domaines où nous ne l'attendons pas, la convoquer permet d'enrichir la compréhension du monde grec antique.

\section{VINCIANE PIRENNE-DELFORGE}

Vinciane Pirenne-Delforge est historienne. Elle a mené sa carrière au Fonds de la recherche scientifique (FNRS) de la Fédération Wallonie-Bruxelles, tout en enseignant à l'université de Liège, où elle a dirigé l'unité de recherche « Histoire et anthropologie des religions ». Elle est professeure au Collège de France, titulaire de la chaire Religion, histoire et société dans le monde grec antique depuis octobre 2017. 
SOMMAIRE

Introduction

Thomas Römer

Le polythéisme grec comme objet d'histoire

Leçon inaugurale prononcée le jeudi 7 décembre 2017

Vinciane Pirenne-Delforge 
NOTE DE L'ÉDITEUR

Cet ouvrage a été réalisé avec la chaîne d'édition structurée XML-TEI Métopes développée par le pôle Document numérique de la Maison de la recherche en sciences humaines (MRSH) de l'université Caen-Normandie. 


\title{
Introduction
}

\author{
Thomas Römer
}

1 Les Grecs ont-ils cru à leurs mythes ? Tel fut le titre d'un essai publié en 1983 par Paul Veyne ${ }^{1}$ , professeur au Collège de France. Derrière ce titre quelque peu insolite se cachait une réflexion profonde sur la fonction du mythe dans la société grecque ancienne, mais aussi dans nos sociétés contemporaines, qui ont toutes des mythes de fondation et de légitimation. Comment un peuple de philosophes, de géomètres, peut-il raconter des histoires de dieux qui ont une forme humaine ou animale, qui s'unissent à des humains et sont lunatiques et imprévisibles ? Peut-on qualifier de tels récits de « religieux », doit-on les prendre au sérieux et quelles fonctions de tels récits peuvent-ils remplir?

2 La tradition mythologique de la Grèce antique est intrinsèquement liée à la question du lien complexe entre religion, histoire et société dans le monde grec antique. Et ce lien se reflète parfaitement dans l'intitulé de la chaire que Mme Vinciane Pirenne-Delforge inaugure ce soir : « Religion, histoire et société dans le monde grec antique ».

3 Mme Vinciane Pirenne est une excellente spécialiste de l'interaction entre les représentations religieuses, les pratiques religieuses et la société dans le monde de la Grèce antique. Elle s'est d'abord fait connaître par une monographie sur la déesse Aphrodite $^{2}$, dans laquelle elle a montré que l'on ne peut réduire une divinité à la représentation que l'on trouve d'elle chez les grands poètes panhelléniques. Comme c'est d'ailleurs le cas dans le Proche-Orient ancien, les divinités sont d'abord caractérisées par leurs ancrages locaux et particuliers. Dans les sanctuaires grecs, on ne rencontre pas Zeus ou Aphrodite per se. Chaque sanctuaire est consacré à une forme particulière de ces divinités, signifiée par une épiclèse qui détermine une fonction ou un pouvoir précis, comme par exemple l'Aphrodite Ourania ou l'Aphrodite Pandémos. La pratique religieuse d'un lieu doit donc tenir compte de la spécificité d'un panthéon auquel appartient la divinité à laquelle on veut rendre un culte.

4 Mme Pirenne-Delforge est également une référence incontournable dans les études sur Pausanias, un érudit voyageur grec qui a vécu au $\mathrm{II}^{\mathrm{e}}$ siècle de notre ère et dont les écrits ont longtemps été considérés comme les ancêtres de nos guides de voyages. Le livre que Mme Pirenne-Delforge a consacré à Pausanias et à sa description de la Grèce ancienne en dix volumes ${ }^{3}$ montre l'importance de cette œuvre, qui témoigne à la fois de la Grèce de 
son temps, mais aussi du passé, donnant accès à des données aussi diverses que l'urbanisme, l'architecture, l'écosystème, l'organisation et les crises politiques, ainsi que les traditions et les pratiques religieuses. Mme Pirenne-Delforge a intitulé ce livre Retour à la source à cause de l'ambivalence de l'expression. D'une part, le périple de Pausanias dans les différentes régions de la Grèce est un voyage à la source de la culture et des traditions religieuses de son auteur, d'autre part, il s'agit pour l'historien moderne d'exploiter une source privilégiée pour mieux comprendre le monde grec antique. Mme Pirenne-Delforge a ainsi entrepris de comprendre l'apport spécifique de cette œuvre à notre connaissance de la religion grecque antique, en jetant notamment un éclairage nouveau et convaincant sur un aspect essentiel du système religieux des Grecs, à savoir la tension entre le général et le particulier, entre le « panhellénique » et le local.

Vinciane Pirenne-Delforge est également une des meilleures spécialistes de la question du ou des sacrifices grecs. Le terme sacrifice emprunté aux Romains recouvre en effet un acte rituel central du Bassin méditerranéen ainsi que du Proche-Orient ancien, et de bien d'autres régions du monde. La thusia des Grecs est, comme Mme Pirenne l'a illustré dans de nombreuses publications, une réalité complexe, qui ne peut être expliquée sur le seul fondement des témoignages littéraires. Il faut aussi, et même surtout, s'intéresser à la documentation épigraphique, qui fait apparaître la variété des procédures. C'est dans cette perspective qu'elle a piloté un projet intitulé Collection of Greek Ritual Norms, qui rassemble une large part d'inscriptions témoignant de pratiques sacrificielles et purificatoires

6 Je pourrais bien entendu continuer cette énumération des apports de Vinciane Pirenne à l'intelligence de la Grèce antique, mais permettez-moi de terminer en disant qu'elle a répondu dans un article à la question de Paul Veyne citée en ouverture. Elle y a montré, d'une manière magistrale, que les Grecs, à l'exemple d'Hésiode et de Pausanias, peuvent émettre des jugements très critiques et sévères concernant leurs mythes, tout en reconnaissant leurs fonctions pour une communauté dans un contexte donné. L'élection de Vinciane Pirenne-Delforge à la chaire Religion, histoire et société dans le monde grec antique a permis de combiner deux grandes traditions des études grecques au Collège de France: l'approche épigraphique ainsi que l'approche de l'histoire des religions. L'élection de Mme Pirenne-Delforge constitue également un enrichissement important pour l'Institut des civilisations du Collège de France puisque ses recherches ouvriront aussi la voie à un travail comparatiste permettant une collaboration et un dialogue fructueux avec de nombreux collègues.

7 Chère Vinciane, je vous souhaite une cordiale bienvenue au Collège de France et me réjouis, avec tous mes collègues, et tout le public, d'entendre votre leçon inaugurale.

\section{NOTES}

1. Paul Veyne, Les Grecs ont-ils cru à leurs mythes? Essai sur l'imagination constituante, Paris, Seuil, 1983 ; rééd. Points, coll. « Essais », 2014. 
2. Vinciane Pirenne-Delforge, L'Aphrodite grecque. Contribution à l'étude de ses cultes et de sa personnalité dans le panthéon archaïque et classique, Liège/Athènes, Centre international d'étude de la religion grecque antique, 1994 (Kernos, suppl. 4).

3. Vinciane Pirenne-Delforge, Retour à la source. Pausanias et la religion grecque, Liège/ Athènes, Centre international d'étude de la religion grecque antique, 2008 (Kernos, suppl. 20).

\section{AUTEUR}

\section{THOMAS RÖMER}

Professeur au Collège de France, titulaire de la chaire Milieux bibliques 


\section{Le polythéisme grec comme objet d'histoire}

Leçon inaugurale prononcée le jeudi 7 décembre 2017

\section{Vinciane Pirenne-Delforge}

1 Monsieur l'Administrateur,

Messieurs les Pro-recteurs de l'université de Liège,

Monsieur le Ministre,

Chers collègues,

Chers amis,

Mesdames et Messieurs,

2 Le Collège de France enseigne tout - docet omnia, telle est sa devise. Mais ce tout enseigné est susceptible de déclinaisons multiples, en fonction de l'actualité de la recherche. Dans cet esprit, certaines chaires accompagnent clairement l'émergence de nouveaux champs d'investigation. Ce n'est évidemment pas le cas d'un enseignement qui s'enracine dans le monde grec antique. Suis-je dès lors devant vous ce soir en raison de la fidélité de l'institution à une tradition qui remonte à ses origines? Sans doute pour partie. Et la conscience aiguë de cet héritage et de la qualité de ceux qui l'ont porté, doublée de la solennité du lieu et du moment, pourrait arrêter la voix de bien plus valeureux que moi. Mais, au-delà de ma personne, et peut-être même au-delà du respect de la tradition séculaire du Collège, c'est une certaine manière d'explorer le monde grec antique que vous avez souhaité convoquer entre ces murs - ou plutôt reconvoquer, j'y reviendrai. L'intitulé de la chaire en témoigne. Et parce que les mots engagent puissamment ceux qui les choisissent, je commencerai par expliciter les termes qui s'y juxtaposent : religion, histoire, société.

3 En effet, ni la religion ni la société ne font partie comme telles du vocabulaire des Grecs de l'Antiquité. Ce ne sont pas non plus des champs disciplinaires à proprement parler, comme l'est l'épigraphie, qui s'est presque continuellement affichée dans des intitulés de chaires du Collège de France, entre celles de Paul Foucart et de Denis Knoepfler, en passant par les enseignements de Maurice Holleaux et de Louis Robert, ou comme l'est également cette autre discipline qu'est la papyrologie, entrée récemment au Collège de 
France grâce à l'élection de Jean-Luc Fournet. Tant la religion que la société sont des concepts élaborés à des fins opératoires pour questionner le passé, dans la conscience qu'ils nourrissent aussi le langage courant, du moins en Europe occidentale ${ }^{1}$. La tension qui découle de ce premier constat fait partie des enjeux sans doute les plus intéressants, mais aussi les plus sensibles, du métier d'historien: forger sur le passé un discours intelligible aux auditeurs et aux lecteurs d'aujourd'hui sans rien céder, ou en cédant le moins possible, aux déterminismes culturels que recèlent nos outils langagiers et nos cadres mentaux.

4 Cette exigence émerge du troisième terme de l'intitulé, celui d'histoire, dont Paul Veyne affirmait ici-même, en la même circonstance, qu'elle "n'existe que par rapport aux questions que nous lui posons ${ }^{2}$ ». Si tel n'était pas le cas, si l'histoire d'une culture donnée était à l'image d'un continent enfoui à ramener au jour en déblayant mécaniquement les sédiments déposés par les siècles, je ne serais pas devant vous aujourd'hui. Bien des savants plus autorisés que moi auraient déjà soldé les comptes d'un passé strictement et intégralement objectivable. Or les comptes ne seront jamais soldés puisque nous n'aurons jamais fini d'interroger sous d'autres angles les communautés humaines qui nous ont précédés.

5 Si les concepts que j'évoquais il y a un instant sont pour une large part ancrés dans le langage d'aujourd'hui, les questions qui fabriquent l'histoire - en tant que discipline et en tant qu'objet - dépendent elles aussi du présent. Et donc du présent de ceux qui viendront après nous. L'ambition et l'humilité de l'historien sont tout entières nichées dans une telle conviction. Comme le concevait Lucien Febvre, autre grand nom de cette maison, il n'y a d'histoire que du présent ${ }^{3}$. Et je saisis cette occasion pour saluer avec émotion le professeur Jean-Pierre Massaut qui, à l'université de Liège, a passionnément enseigné ces principes à des générations d'étudiants, dont je fus. Il incarnait alors l'école liégeoise de la critique historique, après Léon-Ernest Halkin.

6 C'est donc la religion grecque en tant qu'objet d'histoire, ou encore l'histoire des communautés grecques au prisme de leurs représentations et de leurs pratiques religieuses qu'évoque cet intitulé de chaire - et je reviendrai à la "société " plus avant. Mais, si je souscris au point de vue de Paul Veyne sur les concepts nécessaires à l'intelligibilité historique, comment appréhender la religion? Elle n'existe pas comme un donné invariant : il n'existe que des religions historiquement déterminées, caractérisées par des pratiques et des discours dont l'historien essaie de rendre compte comme de tout autre élément culturel. Cependant, si l'on a le droit, et même le devoir, de définir la spécificité d'un tel objet d'étude, c'est la religion en tant que concept qu'il faut convoquer. Le terme désigne alors un ensemble de représentations et de pratiques qui inscrivent l'humain dans une dimension qui le dépasse et reste inconnaissable. Mais la manière dont cela s'opère et se noue est contingente. Chaque religion appelle une analyse qui prenne au sérieux les mondes imaginaires ainsi constitués, les discours et les actes qu'ils induisent et qui, inversement, contribuent à les construire.

7 En ce sens, la religion grecque antique existe, tout comme la religion romaine ou les religions indo-iraniennes, respectivement enseignées au Collège de France par John Scheid et Jean Kellens. Ces ensembles complexes et labiles que l'on appelle « religions » ne relèvent pas du mirage auquel la déconstruction hypercritique de nos catégories d'analyse les a parfois ramenés. En témoigne aussi le travail de Jean-Pierre Vernant, qui inaugurait ici-même, il y a quarante-deux ans, une chaire d'Étude comparée des religions antiques, tout en réservant à la religion grecque la plus grande part de ses 
enseignements. Au registre des dettes, nombreuses, que j'ai contractées au fil de mon parcours, les travaux de ce grand savant généreux sont consignés en bonne place, comme ceux de Jean Rudhardt, qui officiait à Genève, et de Marcel Detienne ${ }^{4}$, un autre Liégeois dont la légitimité à parler de religion grecque en cette maison, il y a trente ans, eût été incontestablement bien supérieure à la mienne aujourd'hui. Comme vous le savez, les dieux, qu'ils soient grecs ou autres, ne cesseront jamais de se gausser des projets et des ambitions des humains, fussent-ils parfaitement fondés.

J'évoquais, en commençant, l'héritage de ceux qui ont exploré, entre ces murs, la Grèce antique, sa langue, sa littérature, son histoire. Toutefois, ce n'est pas le seul fil qu'il me faut tirer car je m'inscris d'une certaine manière aussi dans la voie ouverte par ceux qui se sont réclamés ici-même de l'histoire des religions depuis 1880. L'intitulé « Histoire des religions ", dans sa généralité, a été assumé par les Réville, père et fils, par Alfred Loisy et plus tard par Henri-Charles Puech. Certes, l'histoire des religions telle que les premiers d'entre eux l'entendaient avait encore fort à faire pour s'affirmer dans une démarche scientifique indépendante de la théologie. La question de l'étude scientifique de la tradition biblique et du christianisme est longtemps restée brûlante. La figure même de Loisy et les circonstances de son arrivée au Collège de France après son excommunication l'attestent à suffisance. L'ambition affichée de parler des religions dans une perspective comparée était une manière de mettre le christianisme à distance et de l'inscrire parmi les religions du monde ${ }^{5}$. La tâche était sensible et Loisy lui-même, pourtant le plus soucieux de promouvoir le comparatisme, continuait d'installer le christianisme au centre de sa réflexion ${ }^{6}$. Qu'en fut-il ensuite? Une telle question est importante et ne relève pas uniquement de l'exercice convenu et inaugural qui consiste à s'enraciner dans le terreau local.

9 Pas plus que celles de Jean-Pierre Vernant ou de John Scheid, voire de Michel Tardieu sur les «Syncrétismes de la fin de l'Antiquité » ou encore de Thomas Römer sur les « Milieux bibliques ", la chaire que vous avez choisi de me confier ne se réclame explicitement, dans son intitulé, de l'«histoire des religions ». D'où vient ce qui pourrait apparaître comme une certaine frilosité ? L'histoire des religions a conquis de haute lutte sa légitimité et je me reconnais sans peine dans les attendus de sa démarche. Mais il s'agit précisément d'une démarche et non d'un champ d'investigation englobant. L'ambition d'inclure toutes les religions dans un ample mouvement interprétatif tel que le rêvait un Friedrich Max Müller au milieu du XIX ${ }^{e}$ siècle est définitivement morte en 1986, l'année du décès de Mircea Eliade. Les hasards du calendrier font que cette année-là a également vu s'éteindre Georges Dumézil, qui a enseigné pendant presque vingt ans dans le cadre d'une chaire de Civilisation indo-européenne au Collège de France. Et à ces hasards s'ajoute l'ironie de certains héritages : nombre d'historiens des religions se reconnaîtront aisément dans l'œuvre de Dumézil, une fois mise à distance la clé d'interprétation trifonctionnelle. En revanche, il est plus délicat de s'approprier la démarche d'Eliade, qui relève d'une approche analogique et mystique de la religion comme essence anhistorique. Or, tout en ayant enseigné longtemps à la section des Sciences religieuses de l'École pratique des hautes études, Dumézil n'était pas officiellement étiqueté comme « historien des religions ", ce qu'était en revanche Eliade qui professait à Chicago.

10 À l'acception de l'histoire des religions que j'assume, avec bien d'autres collègues qui se reconnaissent dans cette démarche ${ }^{7}$, est intimement liée la présence d'un terrain spécifique qui fonde l'analyse. Alors, certes, pour un historien, ce terrain ne peut pas être celui d'une observation anthropologique directe, mais il est celui de questionnements que 
l'histoire des religions partage avec l'anthropologie et que, pour ma part, j'applique au monde grec antique. Mais l'horizon de l'investigation en histoire des religions reste encore et toujours la comparaison : Loisy l'affirmait il y a plus d'un siècle, Dumézil, dès 1938, comparait du comparable en contexte indo-européen, tandis que Detienne, au début des années 2000, préconisait de comparer l'incomparable. La fécondité de l'exercice est aujourd'hui reconnue et la démarche est appliquée dans des contextes d'expérimentation où des chercheurs issus d'horizons divers acceptent de s'asseoir à une même table et de poser ensemble des questions qui puissent en retour enrichir leur propre pratique ${ }^{8}$. Les religions ne sont pas le seul terrain d'une telle entreprise. Mais l'exigence s'en fait probablement sentir d'une façon plus impérieuse encore quand on aborde cette dimension de l'aventure humaine. En cette circonstance inaugurale, je forme le vœu que l'Institut des civilisations du Collège de France devienne un laboratoire privilégié pour accueillir ce type d'expérimentation transversale, qui reste encore trop souvent un vœu pieux. Un « historien des religions » s'inscrit forcément dans une démarche collective et les collègues de l'équipe de recherche Anhima (Anthropologie et histoire des mondes antiques) ici présents peuvent attester que nous avons emprunté de telles voies avec curiosité et passion pendant plusieurs années 9 .

11 Je pars donc d'un terrain spécifique. Celui de la religion grecque ou, pour le dire d'une autre manière, celui du polythéisme grec. Pas plus que celui de religion, le terme de polythéisme n'est neutre car il s'est forgé par contraste. Dès le tout début de notre ère, Philon d'Alexandrie, un philosophe juif, mettait en regard la pluralité des dieux honorés sur les différents territoires de la Méditerranée de son temps et l'unicité exclusive du Dieu de la Bible qu'il commentait en grec. La conception d'un monde divin pluriel était identifiée par lui en tant que polytheia ou polytheon ${ }^{10}$. Les Pères de l'Église écrivant en grec se sont à leur tour emparés de ce vocabulaire, mais les Pères écrivant en latin ont privilégié les termes d'idolâtrie et de paganisme qui ont traversé le Moyen Âge occidental. Avec la redécouverte du grec à la Renaissance, polytheia redevient utilisable et Guillaume Budé, lecteur royal de grec aux tout premiers temps du Collège de France, transposera le terme en latin. Peu de temps après, des auteurs écrivant dans les langues vernaculaires de l'Europe allaient forger le terme de polythéisme $e^{11}$. Il faut toutefois attendre le $\mathrm{xVIII}^{\mathrm{e}}$ siècle, celui des Lumières, pour que polythéisme remplace les termes d'origine latine et neutralise, du moins partiellement, leur héritage polémique. Et si je recours très volontiers au terme de polythéisme, en dépit de tous ses défauts - dont le moindre n'est pas de limiter l'identification des religions de ce type à la pluralité de leurs dieux -, c'est parce qu'il s'est progressivement répandu dans des contextes qui tentaient de prendre au sérieux les religions non chrétiennes. Dès lors, parler de « religion grecque » rencontre l'exigence méthodologique de considérer que le concept de "religion" s'applique, au moins à titre exploratoire et opératoire, à toutes les sociétés humaines; parler de "polythéisme grec" met en évidence une caractéristique essentielle de ce système symbolique (même si elle n'est pas la seule) : la conception d'un monde qu'habitent une multiplicité de dieux. 
Engageons-nous à présent sur ce terrain d'exploration dans sa matérialité documentaire. Car un historien est avant tout un praticien du document, sous toutes ses formes, qui sont potentiellement aussi variées et multiples que le sont les expériences humaines. L'attention portée à tous les types de traces du passé est un impératif en histoire de l'Antiquité dont la documentation a connu l'usure progressive des siècles, mais plus encore les naufrages massifs, qu'ils soient accidentels ou le fruit du choix de conserver tels textes plutôt que tels autres, et ce dès l'Antiquité. Quand il s'agit de religion grecque, les traces en question vont des œuvres les plus abouties des auteurs anciens jusqu'aux ossements d'animaux qui parlent de division sacrificielle, en passant par les règlements épigraphiques, les structures architecturales, les milliers de dédicaces et d'artéfacts de tous types.

C'est la raison pour laquelle l'historien des temps contemporains qui partage ma vie - et à qui je dois tant - fait assurément le même métier que moi, mais qu'il n'y a guère de commune mesure entre nos moissons documentaires respectives. Il n'en reste pas moins que le socle documentaire est encore et toujours au cœur de notre pratique, envers et parfois contre les réflexions théoriques qui ont marqué les sciences humaines ces dernières décennies et dont Roger Chartier a bien montré les abîmes qu'elles pouvaient ouvrir sous les pas des historiens ${ }^{12}$. Dans la pleine conscience que le bord de la falaise qu'il décrit n'est jamais loin, je reste lucidement confiante dans la possibilité de déchiffrer le passé et de transposer cette analyse dans un discours qui, s'il adopte plus que d'autres démonstrations scientifiques une forme littéraire, ne relève pas pour autant de la fiction. Mais travailler sur les religions impose une ascèse particulière puisque : «En histoire des religions comme en amour, la plume glisse facilement ${ }^{13}$.»

Mais c'en est assez à présent de ces considérations générales. Partons pour la Grèce.

Je vous emmène tout d'abord en Épire, dans le Nord-Ouest, à Dodone, dans le vénérable sanctuaire oraculaire de Zeus dont parlait déjà l'Iliade (XVI, 234). C'est dans le paysage grandiose qui caractérise encore le lieu aujourd'hui, qu'un certain Évandros et sa femme

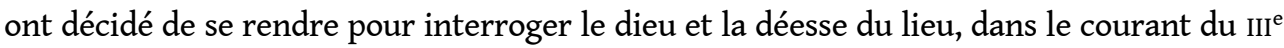
ou du II ${ }^{\mathrm{e}}$ siècle avant notre ère. Ils ont scrupuleusement noté ou fait noter sur une lamelle de plomb la question qui les avait amenés là. Ensuite, ils ont probablement remis l'objet inscrit à l'un des membres du personnel de l'oracle. Une réponse leur a été donnée, dont nous ignorons les modalités de transmission et la teneur. Peut-être Évandros et sa femme ont-ils rapporté chez eux la réponse escomptée, elle aussi inscrite, même si les fouilles attestent que les injonctions divines sur lamelle de plomb ont pu être laissées sur place.

Ce modeste document a été mis au jour sur le site du sanctuaire il y a un siècle et demi, avec une quarantaine d'autres disséminés dans le sol. Des fouilles ultérieures en ont exhumé plusieurs milliers d'exemplaires, sur lesquels la corrosion a exercé son œuvre dans les réserves des musées, où ces objets fragiles attendaient d'être publiés. Des travaux récents ont mis à la disposition de la communauté scientifique celles qui étaient encore 
lisibles parmi ces modestes pièces ${ }^{14}$. Mais leur grande modestie n'a d'égal que les perspectives de tous ordres qu'elles ouvrent aux philologues, aux épigraphistes, aux linguistes, aux dialectologues et aux historiens de la religion grecque.

17 À ce stade, je n'ai encore rien dit de la question posée par le couple de consultants. Ils ont demandé «[...] auquel des dieux ou des héros ou des daimones ils pourraient adresser des prières et sacrifier pour agir au mieux et avec profit, eux-mêmes aussi bien que leur famille, maintenant et pour toujours ${ }^{15}$ ». Nous ne savons rien d'Évandros, ni du lieu ni des circonstances de sa vie. Nous ne connaissons même pas le nom de l'épouse censée avoir posé la question avec lui. En revanche, le libellé de la question est bien connu de ceux qui travaillent sur la divination. Il est comparable à tant d'autres consultations individuelles, mais aussi à des démarches effectuées par des cités. Ainsi les gens de Corcyre, l'actuelle île de Corfou en face de Dodone, ont-ils demandé, eux aussi, «à Zeus Naios et à Dionè à quel dieu ou héros ils doivent sacrifier et adresser des prières pour gouverner au mieux et en toute sécurité leur cité, et pour avoir une bonne et abondante récolte, ainsi que le profit de toute bonne récolte ${ }^{16} »$.

Confrontés aux incertitudes de l'existence, les particuliers et les communautés se tournaient vers les dieux. Le constat serait terriblement banal si une question subsidiaire ne permettait de mettre l'accent sur ce qui est, à mon sens, une préoccupation centrale de l'étude des polythéismes antiques : les particuliers et les communautés se tournaient vers les dieux, certes, mais lesquels? Dans le foisonnement de l'offre divine inhérente à un monde divin pluriel, comment les anciens opéraient-ils leurs choix ? Sur certaines des lamelles de Dodone, on vient de le voir, l'appui de Zeus et de Dionè, sa divine compagne, est sollicité pour le déterminer. Mais les Grecs sont loin d'avoir systématiquement assorti leurs démarches rituelles d'un recours préliminaire à une instance oraculaire. La gestion quotidienne du rapport aux dieux ne requérait pas forcément de tels moyens et bon nombre de gestes traditionnels, précisément parce qu'ils étaient en quelque sorte intégrés, incorporés, n'appelaient pas de délibération préalable. Cependant, pour nous qui tentons de comprendre ce système, la question du choix reste entière pour appréhender le commerce des anciens avec le monde supra-humain.

Cette question se pose à tous les niveaux et à toutes les étapes de la vie des individus et des groupes dont ils font partie, à diverses échelles. Évoquons-en quelques-unes : les cités ont choisi les entités supra-humaines auxquelles elles confiaient leur destinée pour former ce que nous appelons des panthéons locaux; tout au long de leur histoire, elles ont poursuivi cette élaboration en convoquant tel ou tel dieu selon les nécessités du moment, comme le montre l'exemple des gens de Corcyre interrogeant Zeus Naios et Dionè; de plus petites unités politiques locales pouvaient elles aussi disposer d'un panthéon, comme les dèmes de l'Attique en témoignent; des groupes de sociabilité de tous ordres se sont régulièrement constitués autour d'un ou de plusieurs cultes; les maisonnées honoraient divers dieux avec une marge de manœuvre que régulait la pratique ancestrale du groupe familial ; quant aux individus, le cas d'Évandros et de sa femme partant pour Dodone les inscrit dans une même préoccupation, celle qui consiste à bien identifier son interlocuteur divin et à ne pas se tromper d'adresse. 
ne savons pas à qui Évandros et sa femme ont finalement sacrifié pour obtenir les bienfaits qu'ils espéraient. Nous ne savons pas davantage à qui la cité de Corcyre a adressé ses prières pour se mettre à l'abri des périls et assurer son autonomie alimentaire. Mais les uns et les autres ont eu à choisir. Et si leurs sacrifices et leurs prières n'ont pas abouti, gageons qu'ils auront exempté l'oracle de toute responsabilité, en incriminant plutôt une erreur d'aiguillage fondée sur une incompréhension de leur part. Filant quelque peu la métaphore ferroviaire, j'ajouterai que les aiguillages sont nombreux en contexte polythéiste. Mais qui dit réseau ferroviaire dit aussi la rigueur des tracés sur un terrain à chaque fois déterminé par la topographie locale. C'est une manière parmi d'autres de se représenter le polythéisme grec: un ensemble structuré de voies possibles dans un contexte particulier, voire spécifique, pour atteindre toutes sortes d'objectifs.

Et, quittant le domaine ferroviaire tout en conservant l'idée du réseau, chaque entité supra-humaine de cet ensemble pluriel peut elle aussi être envisagée comme un réseau polycentrique complexe puisque, grâce à Jean-Pierre Vernant, nous avons compris que les dieux grecs étaient des puissances et non des personnes ${ }^{17}$. La métaphore du réseau donne assurément matière à penser les puissances divines et le polythéisme ${ }^{18}$. Cependant, quel qu'en soit le pouvoir d'évocation, une métaphore ne pourra jamais tenir lieu de démonstration. C'est non seulement en posant des questions précises à la documentation, fût-elle aussi modeste qu'une lamelle de plomb mise au jour à Dodone, mais aussi en identifiant les questions peu ou non pertinentes qui ont parasité l'étude de la religion grecque - et continuent de le faire - que l'on peut espérer voir progresser notre compréhension de la composante du monde grec antique qui reste sans doute la plus exotique à saisir.

Car la religion grecque antique a bien quelque chose d'exotique. À tel point que, pendant des siècles, il s'est trouvé des admirateurs de cette prestigieuse culture considérée comme fondatrice pour s'interroger sur une telle curiosité : comment les inventeurs de l'histoire, de la philosophie, de la géométrie, de la politique, de la démocratie, comment les contemporains d'un Homère, d'un Eschyle, d'un Platon ou d'un Aristote avaient-ils pu adhérer à un système religieux aussi fruste et approximatif, une fois que l'on avait renoncé à y trouver quelque prescience de la révélation? La question est tout sauf innocente, elle est tout sauf dépassée car elle continue de travailler en profondeur la réflexion sur la naissance de la pensée rationnelle et philosophique dans les cités grecques de l'Ionie, sur la côte de la Turquie actuelle, là où virent le jour bon nombre de ceux qu'on appelle les " philosophes présocratiques".

La religion grecque est le caillou dans la chaussure de ceux qui, d'une manière ou d'une autre, continuent de penser le $\mathrm{v}^{\mathrm{e}}$ siècle avant notre ère comme l'irréversible point de basculement vers le logos, à savoir l'exercice d'une pensée rationnelle débarrassée du muthos, le mythe. L'image que le miroir de cette Grèce-là tend à notre modernité a quelque chose d'irrésistible. Mais il est nécessaire de résister, et cela passe notamment par une meilleure compréhension des entrelacements inextricables de ces catégories que les chercheurs modernes ont érigées en principes d'opposition: le mythe, la raison, la raison du mythe, la raison des mythes, et les raisons ou non d'y croire. Je reconnais volontiers jouer ainsi sur les mots, mais le jeu n'est pas gratuit: il a tout le sérieux des travaux qu'un chercheur comme Claude Calame consacre à ces questions depuis des années ${ }^{19}$ en faisant partie de cette "résistance » que j'évoquais et que je salue, tout comme les travaux d'André Motte que je remercie publiquement de son indéfectible soutien depuis plus de trente ans. Il y a bien longtemps qu'il a compris que les dieux 
n'avaient pas été ostracisés de la pensée des présocratiques, de celle de Platon lui-même, et qu'une conception rigide des "Lumières à la grecque " produisait d'aveuglants anachronismes.

Sans aller toujours jusqu'à l'aveuglement, une certaine myopie risque de frapper notre regard d'Européens du xxI ${ }^{e}$ siècle dès que l'on arrive en pays polythéiste. Car les dieux sont là et cette présence a de nombreuses conséquences dans toute une série de domaines où l'on n'est pas nécessairement préparé à les voir. Plus encore, cette place des dieux ne peut se comprendre qu'en relation étroite avec la vision globale que les Grecs avaient du monde dont ils faisaient partie, en ses diverses composantes. Vous aurez peut-être remarqué mon insistance à parler des figures supra-humaines que sont les dieux, et non de surnature ou de surnaturel. Ce n'est évidemment pas un hasard. Tant les dieux que les hommes participent d'un cosmos où ne se marque pas de coupure ontologique radicale. La frontière qui passe entre les humains et les dieux relève globalement d'une différence de qualité de l'être, d'intensité dans l'être plutôt que d'une incommensurable altérité. Audelà d'une certaine manière d'entrer en relation avec les dieux et d'exprimer leur action, la forme humaine que les Grecs leur prêtaient volontiers tient aussi à cette immanence du divin. Et la catégorie des héros, si particulière à la culture grecque, y trouve une partie de son enracinement.

Les dieux sont là, disais-je, les héros aussi. Dès lors, dans la définition d'une cité grecque, une polis, on trouve les traits suivants : tout d'abord, un groupe d'hommes qui forme une société maîtresse de son destin sur un territoire particulier et limité, clairement identifiable parmi les autres cités; ensuite, des dieux et des héros qui partagent ce territoire et contribuent même à le structurer; enfin, un ensemble de traditions, de coutumes ancestrales, bref ce que nous appelons une « culture ». J'ai ainsi placé les dieux et les héros sur le même plan que les autres caractéristiques de la cité plutôt que de les immerger dans la seule référence aux traditions culturelles. Cela ne signifie pas que je veuille abstraire la religion des autres composantes de ce type. Je souhaite ainsi en souligner l'importance dans la définition même des cités grecques à quelque moment de leur histoire que ce soit. Les dieux sont parties prenantes du tissu social de ces petites communautés politiques dispersées dans le monde grec. La société - et j'y arrive enfin est précisément la notion qui nous permet d'agréger les contraintes matérielles auxquelles sont soumises les cités et les rapports de force qui s'y déploient, toutes choses que l'attention portée au monde supra-humain permet d'interroger autrement.

Un tel constat sur ce qui fait la cité engage immédiatement les questions d'autorité et de pouvoir. À cet égard, on a depuis longtemps affirmé que, dans ce monde-là, celui de la polis, ou plutôt des poleis, l'absence d'une transcendance divine absolue, d'une révélation sacrée et unifiée, et de toute classe sacerdotale avait permis l'émergence d'un espace public commun à tous, où une expérimentation politique particulière avait pu prendre place. Jean-Pierre Vernant a laissé de magnifiques pages sur la neutralisation du kratos, de ce " pouvoir » auquel tous les citoyens participent sans qu'aucun ne soit plus en mesure de se l'approprier ${ }^{20}$. La formulation même des décisions collectives ( La cité a décidé [... ]» ou «Il a plu au Conseil et au Peuple [...]») montre que l'autorité est tout aussi 
immanente que les dieux, et que tout type de décision appartient aux citoyens qui s'assemblent pour la prendre.

Toutefois, il ne suffit pas de subordonner la création de cet espace commun aux seules absences et aux seuls manques qui caractériseraient la religion grecque. Cette détermination par défaut ne rend pas suffisamment justice au fonctionnement du polythéisme en sa version grecque, ni à la politique telle que les Grecs en ont poussé l'autonomie à un degré remarquable. L'imbrication étroite de ces deux sphères de l'activité des cités ne relève pas forcément de ce qui manquerait à la religion grecque, mais bien de ce qu'elle est en tant que système polythéiste, c'est-à-dire un ensemble fondamentalement pluriel, flexible, adaptable, et où les voies de l'autorité sont plurielles elles aussi, en fonction du niveau de décision concerné ${ }^{21}$. Beaucoup reste à faire pour appréhender le polythéisme en ce qu'il est et non seulement en contraste avec ce qu'il n'est pas.

Or, à cet égard, des lignes de force peuvent être tracées, dans la perspective d'ouverture d'un programme d'enseignement. Ces lignes de force s'enracinent dans les voies d'investigation que je poursuis depuis longtemps.

Plusieurs d'entre elles se dessinent sur l'arrière-plan particulier de la structuration du monde grec tout au long de son histoire. En effet, à la fin de la période classique, au IV ${ }^{\mathrm{e}}$ siècle avant notre ère, ce monde comptait un peu plus de mille cités, réparties sur les bords de la mer Égée, sur les côtes de la mer Noire et celles de la Méditerranée occidentale. Il comptait aussi d'autres formes d'organisation politique, chevillées à un polythéisme également foisonnant. Un tel ensemble ne formait pas une unité politique avant l'émergence des royautés hellénistiques et l'arrivée de Rome. Quant à l'unité culturelle, elle parait en revanche ne pas faire de doute. Les Grecs semblent avoir assumé dans un même mouvement l'hellénisme partagé et une myriade de centres politiques. Leur culture s'étendait largement sur les rivages des mers, tandis que l'identité politique restait limitée aux frontières étroites de leurs multiples cités, à tel point qu'un Grec arrivant dans une polis différente de la sienne était bel et bien un étranger. Il n'avait donc pas accès, sauf à se faire représenter par un citoyen, aux institutions civiques d'une autre cité que la sienne. Mais ce tableau contrasté entre fractionnement politique et unité culturelle doit être fortement nuancé, notamment quand il s'agit de religion. En effet, la prémisse qui le porte est discutable en ce qu'elle distingue institutions politiques et activités religieuses. Or ce même Grec que l'on vient de mettre en scène dans ses pérégrinations vers une autre cité pouvait tout autant se voir refuser l'accès à l'un ou l'autre sanctuaire de ladite cité au motif qu'il était étranger ${ }^{22}$. Cependant, tandis qu'il reconnaissait assurément, dans cette cité différente de la sienne, les principes généraux $\mathrm{du}$ fonctionnement de sa propre polis, le nom des divinités du lieu devaient également jouer à son oreille une musique rendue familière par la récitation des épopées, et les sacrifices accomplis pour honorer les dieux devaient déployer sous ses yeux des gestes connus. Les gradations sont donc multiples dans l'évaluation de ce qui rapprochait et séparait les Grecs à toutes les échelles de la vie des cités et des relations entre elles.

Le cas des fondations coloniales est symptomatique de cet état de fait. Quand un groupe s'apprêtant à lever les voiles vers l'inconnu était composé de Grecs provenant de cités 
différentes, l'établissement des us et coutumes - les nómaia - de la nouvelle implantation n'allait sans doute pas de soi, même si nous manquons d'informations à ce sujet ${ }^{23}$. Mais la synthèse était finalement possible, dans une gestion pragmatique entre le général et le particulier. Explorer le polythéisme grec de ce point de vue permet d'affiner notre compréhension de ce qu'était le monde grec antique, entre unité et diversité, à chaque étape de son histoire, selon des articulations qui se redéfinissent sans cesse.

Sur cet arrière-plan, une première question concerne la relation entre les traditions narratives qui parlent abondamment des dieux et des héros et les cultes des cités qui honorent ces mêmes dieux et héros. En d'autres termes: que reste-t-il d'Artémis, d'Apollon ou d'Aphrodite, de Zeus, d'Héra ou d'Hermès quand ces divinités passent des œuvres épiques, qui faisaient partie d'un patrimoine largement partagé, aux sanctuaires des centaines de cités où elles recevaient un ou plusieurs cultes? Une autre question concerne les gestes posés pour honorer ces dieux : sur quel fondement étaient édictées les normes rituelles présentes à tous les niveaux de l'hommage à leur rendre ? Dans l'un et l'autre cas, que ce soit la représentation du divin ou les normes rituelles, c'est la multiplicité qui prévaut au plan local. Dans les cités, les dieux reçoivent des titres cultuels qui expriment les diverses potentialités de leur action dans le monde, les héros locaux s'ancrent dans le territoire par dizaines. Quant aux rituels, ils explorent tous les ressorts d'un langage sacrificiel qui permet d'entrer en communication avec les dieux, tout en parlant beaucoup des hommes.

Mais au-delà du chatoiement des motifs singuliers, il existe une trame sous-jacente, une sorte d'" air de famille" qui implique la reconnaissance réciproque et les référents partagés. Quand la cité de Corcyre allait interroger l'oracle de Dodone, ainsi qu'on l'a vu plus haut, ou même la cité d'Athènes, pourtant située à plusieurs centaines de kilomètres du site, cela signifie que le Zeus de Dodone était un interlocuteur pour l'une comme pour l'autre. Et le cas de l'Apollon de Delphes est plus clair encore. On pourrait, certes, me rétorquer que ces sanctuaires revêtaient une dimension particulière, celle du panhellénisme, comme c'était le cas aussi de celui d'olympie. Mais précisément! Le fait que de tels lieux aient émergé dans le paysage religieux des cités dès la période archaïque atteste que la tension entre le général et le particulier est inhérente au système polythéiste. Ce dernier se définit sur le terrain en même temps que naissent les communautés politiques et évolue avec elles jusqu'en plein Empire romain.

Souvenons-nous qu'au temps des commencements se cristallisent aussi, avec l'adoption de l'écriture alphabétique, les traditions épiques dont seules des bribes nous sont parvenues, fussent-elles aussi sublimes que l'Iliade, l'Odyssée ou la Théogonie. D'où la question que je viens de poser et que je précise : que reste-t-il, par exemple, de la figure d'Héra quand on passe de l'Iliade à la cité d'Argos ou à celle de Corinthe? Le choix de cette déesse n'est pas indifférent puisque ma collègue Gabriella Pironti et moi-même avons passé quelque temps à rendre à Héra ce qui appartient à Héra. Au-delà de ce qui pourrait apparaître comme une énième monographie sur un dieu grec, nous avons conduit une expérimentation du polythéisme dont les implications méthodologiques dépassent cette seule figure divine ${ }^{24}$. Il s'agissait de comprendre comment la mégère de l'Iliade, l'épouse en colère et la belle-mère pénible s'articulaient à la figure tutélaire de cités comme Argos ou Samos. Nous avons combattu une certaine myopie ambiante telle que je l'évoquais plus haut : celle qui méconnaît les liens étroits qui se nouent entre les traditions narratives et les cultes, et qui crédite les Grecs d'une mythologie divertissante en contraste présumé avec leurs démarches rituelles. 

nómos - les nómima, les nómaia, je l'ai dit, les nomizómena et les nómoi, un terme qui en viendra à désigner les lois elles-mêmes. Le champ sémantique du nómos relève étymologiquement de la division et de la répartition. C'est au cœur d'un tel ensemble que se détermine ce que nous appelons une "culture", dans le sens de la couleur et de la saveur spécifique des pratiques et des formes de pensée d'un groupe humain. Les déclinaisons en sont nombreuses et elles incluent notamment - mais pas exclusivement ce que nous appelons "religion", dans la tension entre le général et le particulier évoquée plus haut. Dans ce monde grec, que le Collège de France choisit de maintenir au 
programme de ses enseignements, les témoignages les plus nombreux de ces informations sur la vie religieuse sont livrés par les inscriptions qui prescrivent la tenue de rituels et en déterminent les conditions. La moisson épigraphique est continue à cet égard et c'est de là que sont venues, ces dernières années, les nouveautés les plus étonnantes et, dès lors, les questionnements parmi les plus féconds pour notre compréhension du polythéisme grec à toutes les périodes de son histoire.

Prenons l'exemple du sacrifice, dont la compréhension s'est indubitablement affinée en quarante ans par une lecture des inscriptions attentive aux détails de la manipulation des carcasses animales et l'analyse de plus en plus fine des ossements de ces mêmes animaux sur les sites archéologiques. Le traitement des os et de la viande est un remarquable révélateur à la fois de la communication que les sacrifiants mettaient en place avec leurs dieux et de la hiérarchie sociale qu'ils affirmaient dans la distribution des parts. L'accès à la viande engage la question du statut des acteurs, qu'ils soient hommes, femmes, citoyens, métèques, prêtres ou magistrats: l'interprétation fine et approfondie des inscriptions permet d'élucider des problèmes que l'interprétation des seuls textes dits « littéraires» a affrontés en appliquant des grilles d'analyse qui leur étaient propres ${ }^{27}$. C'est pourquoi le projet intitulé Collection of Greek Ritual Norms, dont le site web a été ouvert il y a quelques mois ${ }^{28}$, fait partie des outils que j'ai l'intention de continuer à forger dans ce nouvel atelier, avec l'aide des chercheurs et des collaborateurs que j'ai pu m'associer.

Le moment inaugural de la chaire que je vais désormais occuper arrive tout doucement à son terme et je n'ai encore rien dit de l'inscription d'un tel enseignement dans les enjeux du présent. Il y a un siècle, Alfred Loisy affirmait que «l'étude impartiale et sereine du passé religieux de l'humanité n'est pas indifférente à la solution des graves problèmes qui s'agitent au sein de la société contemporaine ${ }^{29} \%$. Il pensait évidemment à la crise moderniste traversée par l'église catholique romaine qui l'avait frappé d'excommunication. Aujourd'hui, à d'autres échelles, des problèmes graves continuent d'agiter notre monde et les religions font quotidiennement irruption dans l'actualité. Mais si l'histoire des religions n'est pas indifférente aux problèmes, elle n'a pas l'ambition d'être leur solution. Elle est un instrument de mise à distance critique des religions et offre une réflexion continue sur ce que l'on entend par « religion ».

Elle met ainsi à la disposition de tous « ce que peut l'histoire » - pour paraphraser le titre de la leçon inaugurale de Patrick Boucheron. Dans le cadre de la chaire que vous me confiez, "ce que peut l'histoire " est de contribuer à une meilleure appréhension des voies multiples que des humains vivant en société ont empruntées pour s'inscrire dans un monde conçu comme enchanté. C'est peu sans doute, en regard de l'ampleur des défis d'aujourd'hui. Mais c'est beaucoup si l'on mène cette enquête dans le respect des différences et des spécificités, ainsi que John Scheid, que je remercie de sa confiance et de son soutien, l'a régulièrement montré en étudiant la religion romaine. 
41 À la fin de la leçon inaugurale de sa chaire d'Antiquités nationales, en décembre 1984, Christian Goudineau saluait les archéologues de terrain en exprimant l'impression quelque peu gênante de contempler, depuis le jardin d'Éden, ceux qui travaillent à la sueur de leur front. Je pourrais facilement faire mienne cette impression en l'adaptant au paysage de la recherche tel qu'il s'offre à nous aujourd'hui. J'ai le sentiment du privilège insigne de rallier le jardin d'Éden, en regard des restrictions et des réformes qui frappent les institutions d'enseignement supérieur, en regard de la fragilité des jeunes chercheurs précarisés, en regard de l'inflation des tâches de toutes sortes, notamment les évaluations, qui s'abattent sur mes collègues enseignants-chercheurs, vous tous qui m'accompagnez aujourd'hui.

La recherche a besoin de moyens, bien sûr, mais elle a aussi besoin de calme, elle a besoin de temps, de liberté et de confiance; elle a besoin des conditions favorables dont je sais que cette maison les crée pour ceux qui l'habitent, mais dont la conception entrepreneuriale des universités est en train de priver beaucoup de ses acteurs de première ligne. Le Collège de France est assurément un lieu exceptionnel. Mais il s'enracine dans le terreau fertile de centaines de centres de recherche et de laboratoires. Et l'avenir sera forcément partagé, quel qu'il soit.

En m'accueillant parmi vous, chers collègues, vous me témoignez une confiance dont j'espère être digne. J'espère l'être tout autant des institutions qui ont créé les conditions de mon parcours scientifique, à savoir le Fonds de la recherche scientifique de la Fédération Wallonie-Bruxelles et l'université de Liège. Je souhaite honorer ainsi toutes les collaborations que j'ai pu nouer au fil de ces années avec des collègues français car j'ai conscience d'être accueillie dans l'un des établissements les plus prestigieux de la République. Et pour une Wallonne, une Liégeoise de surcroît, l'appel de la France a une saveur toute particulière.

En attendant l'avenir, dont nous aurons collectivement à nous charger, j'arrive au terme de cette leçon inaugurale, dont je vous remercie d'avoir été les témoins.

\section{NOTES}

1. Le Tour du monde des concepts, orchestré par Pierre Legendre (Paris, Fayard et Institut d'études avancées de Nantes, coll. "Poids et mesures du monde», 2014), inclut «religion» et «société » parmi les neuf concepts choisis et mis à l'épreuve de neuf langues non occidentales.

2. Paul Veyne, L'Inventaire des différences, Paris, Seuil, 1976, p. 9.

3. Il ne s'agit pas d'une citation littérale, mais d'une considération récurrente que l'on retrouve sous sa plume dans les Annales: e.g. Annales d'histoire économique et sociale, vol. 1, 1929, p. 99 : «[...] nous ne séparerons jamais de l'étude du passé l'examen attentif du présent »; Annales. Économies, sociétés, civilisations, vol. 1, 1946, p. 8 : «[...] l'Histoire posant des problèmes au passé, en fonction des besoins présents de l'Humanité ». 
4. Jean-Pierre Vernant, Euvres, Paris, Seuil, 2007 ; Jean Rudhardt, Opera inedita, Liège, Presses universitaires de Liège, 2008 (Kernos, suppl. $n^{\circ} 19$ ); Marcel Detienne, Les Grecs et nous. Une anthropologie comparée de la Grèce antique, Paris, Perrin, 2005, auxquels je pourrais ajouter les travaux de Philippe Borgeaud, Pierre Brulé, Claude Calame, et tant d'autres aussi hors de la francophonie, dont Walter Burkert, Fritz Graf, Robert Parker, Henk Versnel.

5. Alfred Loisy, Mémoires pour servir à l'histoire religieuse de notre temps, t. III (1908-1927), Paris, Nourry, 1931, p. 101-102.

6. Ibid., p. 102.

7. À ce sujet, voir le bel article de Philippe Borgeaud, "Qu'est-ce que l'histoire des religions? », Equinoxe, $\mathrm{n}^{\circ} 21,1999$, repris dans Exercices d'histoire des religions. Comparaison, rites, mythes et émotions, textes réunis et édités par Daniel Barbu et Philippe Matthey, Leyde, Brill, 2016, p. 47-68.

8. Marcel Detienne, Les Grecs et nous, op. cit., p. 63-87, 145-176.

9. Ce fut l'aventure «FIGVRA. Représentation du divin dans les mondes grec et romain » pilotée par Nicole Belayche. Sept ouvrages collectifs sont sortis de cet atelier.

10. E.g. De Opificio, 172 ; De Ebrietate, 110 ; De Mutatione nominum, 205.

11. Francis Schmidt, "Naissance des polythéismes (1624-1757)», Archives de sciences sociales des religions, $\mathrm{n}^{\circ} 59,1985, \mathrm{p} .77-90$ : cet article reste fondamental sur ce processus (hormis pour l'apport de Budé qu'il n'a pas abordé : e.g. Opera omnia, t. I, 18, 33 ; 137, 25 ; 143,20 ; etc.).

12. Roger Chartier, Au bord de la falaise. L'histoire entre certitudes et inquiétude, Paris, Albin Michel, 2009.

13. Paul Veyne, «Inviter les dieux, sacrifier, banqueter. Quelques nuances de la religiosité gréco-romaine ", Annales. Histoire, sciences sociales, vol. $55, \mathrm{n}^{\circ} 1,2000, \mathrm{p} .3-42$, citation à la page 32.

14. Éric Lhôte, Les Lamelles oraculaires de Dodone, Genève, Droz, 2006; Sotiros Dakaris,

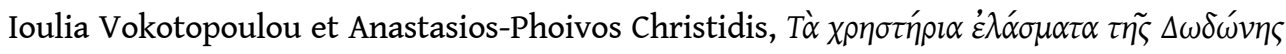

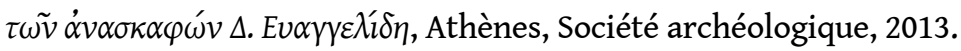

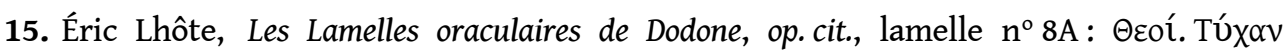

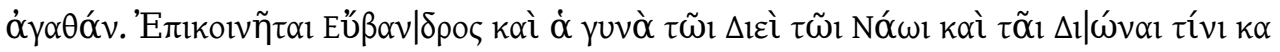

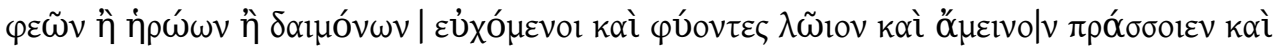

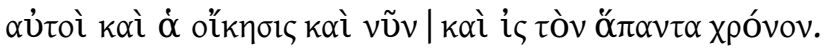

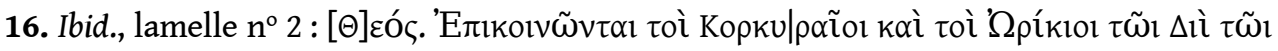

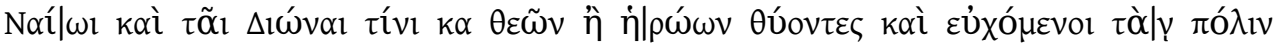

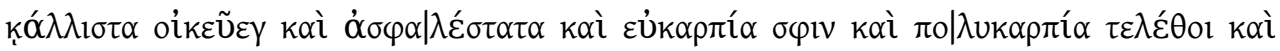

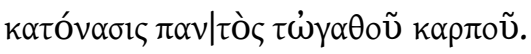

17. Jean-Pierre Vernant, Cuvres, op. cit., p. 567.

18. Sur ce point comme sur d'autres, les travaux de Gabriella Pironti sont essentiels, notamment Entre ciel et guerre. Figures d'Aphrodite en Grèce ancienne, Liège, Presses universitaires de Liège, 2008 (Kernos, suppl. $\mathrm{n}^{\circ}$ 18).

19. Dernièrement : Qu'est-ce que la mythologie grecque ?, Paris, Gallimard, 2015. 
20. Jean-Pierre Vernant, CEuvres, op. cit., p. 233-234, 436-485. Cf. aussi Marcel Detienne, Les Maîtres de vérité dans la Grèce archaïque [1967], Paris, Pocket, 1994, p. 131-157 (« Le procès de laïcisation »).

21. À ce sujet, voir Robert Parker, On Greek Religion, Ithaca, Cornell University Press, 2011, p. 1-63.

22. Le cas assurément le plus célèbre est celui de Cléomène de Sparte dont Hérodote raconte qu'il se vit refuser l'entrée du sanctuaire d'Athéna Polias sur l'acropole d'Athènes par la prêtresse du lieu (Hérodote, V, 72). Cf. Robert Parker, Cleomenes on the Acropolis: An Inaugural Lecture Delivered before the University of Oxford on 12 May 1997, Oxford, Clarendon Press, 1998.

23. Irad Malkin, " "Tradition" in Herodotus: The foundation of Cyrene ", in Peter Derow et Robert Parker (dir.), Herodotus and his World, Oxford, Oxford University Press, 2003, p. 153-170, en particulier p. 164-170.

24. Vinciane Pirenne-Delforge et Gabriella Pironti, L'Héra de Zeus. Ennemie intime, épouse définitive, Paris, Les Belles Lettres, 2016.

25. Bernard de Fontenelle, De l'origine des fables [1724], in : CFuvres complètes, t. III, Paris, Fayard, 1989, p. 202.

26. Sur l'application d'une approche cognitiviste à la religion grecque, voir la synthèse de Jennifer Larson, Understanding Greek Religion, New York, Routledge, 2016. Les inconsistencies sont au cœur de la réflexion d'Henk Versnel dans plusieurs livres absolument essentiels, dont le dernier s'intitule Coping with the Gods: Waymard Readings in Greek Theology, Leyde, Brill, 2011.

27. Sur l'apport de la zoo-archéologie à la compréhension du sacrifice grec, les travaux de Gunnel Ekroth sont essentiels. Voir par exemple « Meat, men and gods. On the division of the animal victim at Greek sacrifices », in A. P. Matthaiou et I. Polinskaya (dir.), Mikpó $\varsigma$

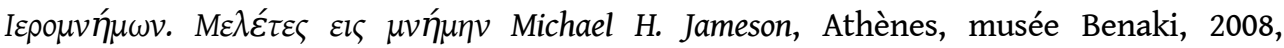
p. 259-290.

28. http://cgrn.ulg.ac.be. Voir Jan-Mathieu Carbon et Vinciane Pirenne-Delforge, «Beyond Greek "Sacred Laws" ", Kernos, n 25, 2012, p. 163-182. L'idée de ce projet est née à Rennes, sous l'impulsion de Pierre Brulé. J'espère qu'il s'y reconnaît, même si les prémisses en ont été profondément modifiées.

29. Alfred Loisy, Mémoires pour servir à l'histoire religieuse de notre temps, op. cit., p. 102.

\section{AUTEUR}

\section{VINCIANE PIRENNE-DELFORGE}

Professeure au Collège de France, chaire Religion, histoire et société dans le monde grec antique 